\title{
Safety and immunogenicity of inactivated SARS-CoV-2 vaccine in high-risk occupational population: a randomized, parallel, controlled clinical trial
}

Yongliang Feng ${ }^{1,2 \dagger}$, Jing Chen ${ }^{3,4 \dagger}$, Tian Yao ${ }^{1,2 \dagger}$, Yue Chang ${ }^{1,2}$, Xiaoqing $\mathrm{Li}^{3,4}$, Rongqin Xing ${ }^{5}$, Hong Li3,4, Ruixue Xie ${ }^{1,2}$, Xiaohong Zhang ${ }^{3,4}$, Zhiyun Wei ${ }^{3,4}$, Shengcai Mu ${ }^{3,4}$, Ling Liu ${ }^{3,4}$, Lizhong Feng ${ }^{3,4^{*}}$ and Suping Wang ${ }^{1,2^{*}}$ (D)

\begin{abstract}
Background: Severe acute respiratory syndrome coronavirus 2 (SARS-CoV-2) infection and the resulting coronavirus disease 2019 (COVID-19) have a substantial burden on health-care systems around the world. This is a randomized parallel controlled trial for assessment of the immunogenicity and safety of an inactivated SARS-CoV-2 vaccine, aiming to determine an appropriate vaccination interval of the vaccine for high-risk occupational population.

Methods: In an ongoing randomized, parallel, controlled phase IV trial between January and May 2021 in Taiyuan City, Shanxi Province, China, we randomly assigned the airport ground staff and public security officers aged 18 to 59 years to receive two doses of inactivated SARS-CoV-2 vaccine at 14 days, 21 days, or 28 days. The serum neutralizing antibody to live SARS-CoV-2 was performed at baseline and 28 days after immunization. Long-term data are being collected. The primary immunogenicity endpoints were neutralization antibody seroconversion and geometric mean titer (GMT) at 28 days after the second dose. Analysis of variance (ANOVA), chi-square, and logistic regression analysis were used for data analysis.

Results: A total of 809 participants underwent randomization and received two doses of injections: 270, 270, 269 in the $0-14,0-21$, and 0-28 vaccination group, respectively. By day 28 after the second injection, SARS-CoV-2 neutralizing antibody of GMT was 98.4 (95\% Cl: 88.4-108.4) in the 0-14 group, which was significantly lower compared with 134.4 (95\% Cl: 123.1-145.7) in the 0-21 group ( $P<0.001$ vs $0-14$ group) and 145.5 (95\% Cl: 131.3-159.6) in the 0-28 group ( $P<0.001$ vs $0-14$ group), resulting in the seroconversion rates to neutralizing antibodies (GMT $\geq 16$ ) of $100.0 \%$ for all three groups, respectively. The intention-to-treat (ITT) analysis yielded similar results. All reported adverse reactions were mild.
\end{abstract}

\footnotetext{
*Correspondence: 1508717672@qq.com; supingwang@sxmu.edu.cn

${ }^{\dagger}$ Yongliang Feng, Jing Chen, and Tian Yao are co-first authors

${ }^{1}$ Department of Epidemiology, School of Public Health, Shanxi Medical University, 56 Xinjian South Road, Taiyuan 030001, Shanxi Province, China

${ }^{3}$ Shanxi Provincial Center for Disease Control and Prevention, 8 Xiaonanguan Street, Taiyuan 030012, Shanxi Province, China

Full list of author information is available at the end of the article
}

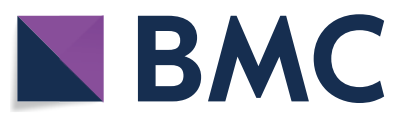

(c) The Author(s) 2021. Open Access This article is licensed under a Creative Commons Attribution 4.0 International License, which permits use, sharing, adaptation, distribution and reproduction in any medium or format, as long as you give appropriate credit to the original author(s) and the source, provide a link to the Creative Commons licence, and indicate if changes were made. The images or other third party material in this article are included in the article's Creative Commons licence, unless indicated otherwise in a credit line to the material. If material is not included in the article's Creative Commons licence and your intended use is not permitted by statutory regulation or exceeds the permitted use, you will need to obtain permission directly from the copyright holder. To view a copy of this licence, visit http://creativecommons.org/licenses/by/4.0/. The Creative Commons Public Domain Dedication waiver (http://creativeco mmons.org/publicdomain/zero/1.0/) applies to the data made available in this article, unless otherwise stated in a credit line to the data. 
Conclusions: Both a two-dose of inactivated SARS-CoV-2 vaccine at 0-21 days and 0-28 days regimens significantly improved SARS-CoV-2 neutralizing antibody level compared to the $0-14$ days regimen in high-risk occupational population, with seroconversion rates of $100.0 \%$.

Trial registration: Chinese Clinical Trial Registry, ChiCTR2100041705, ChiCTR2100041706. Registered 1 January 2021, www.chictr.org.cn.

Keywords: COVID-19, Inactivated SARS-CoV-2 vaccine, Immunogenicity, Safety, High-risk occupational population, Randomized controlled trial

\section{Graphical Abstract}

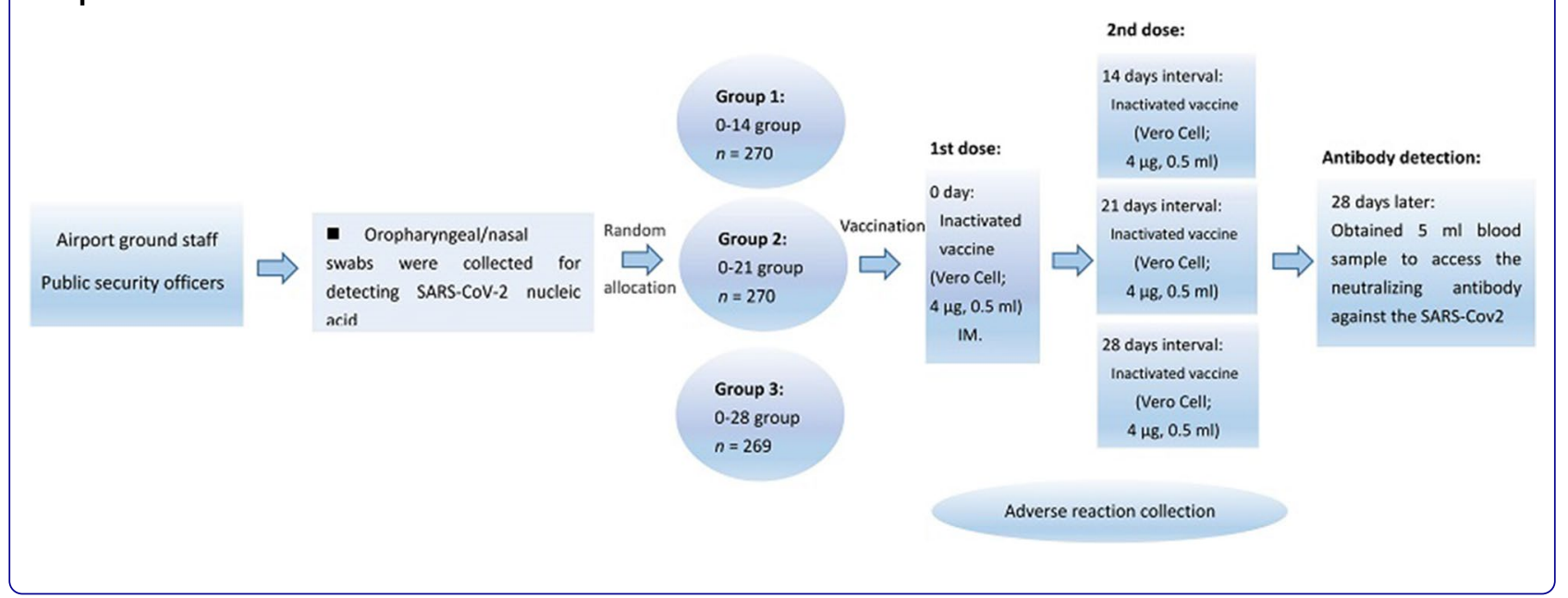

\section{Background}

The ongoing pandemic of coronavirus disease 2019 (COVID-19) induced by severe acute respiratory syndrome coronavirus 2 (SARS-CoV-2) has led to an unprecedented global public health crisis. Globally, as of 26 October 2021, more than 243 million cases of SARS$\mathrm{CoV}-2$ infection and more than 4.9 million deaths have been reported [1]. SARS-CoV-2 appears to undergo more rapid transmission and variation $[2,3]$, and due to the lack of standard treatments, a safe and effective vaccine against COVID-19 is urgently needed to prevent the resurgence of the epidemic.

Inactivated viruses have been traditionally used for vaccine development and such vaccines have been found to be safe and effective for the prevention of diseases caused by viruses like influenza virus and poliovirus $[4,5]$. Their long history of use confers some advantages, such as well-developed and mature manufacturing processes, and ease of scaling up production and storage. Inactivated SARS-CoV-2 vaccines have been confirmed to induce high levels of neutralizing antibody titers in mice, rats, guinea pigs, rabbits, and nonhuman primates to provide protection against SARS-CoV-2 [6-8]. Moreover, the results of previous clinical trials on the inactivated SARS-CoV-2 vaccines conducted in several countries showed good neutralizing antibody responses and efficacy against disease caused by COVID-19 [9-13]. To date, two inactivated SARS-CoV-2 vaccines manufactured by the Beijing Institute of Biological Products/Sinopharm (China) and Sinovac Life Sciences/CoronaVac (China) have received conditional marketing approval from China National Medical Products Administration and have been placed on WHO's Emergency Use Listing $[14,15]$.

Previous studies $[12,13,16-20]$ have shown that the three immunization programs $(0,14$ procedure, 0,21 procedure or 0,28 procedure) induce varying degrees of immune effect, but the optimal interval of injections remains unclear. Furthermore, there is lack of studies on the immunogenicity and safety of inactivated SARS-CoV-2 vaccine in high-risk occupational population. The airport ground staff and public security officers, as front-line workers to respectively responsible for ensuring the operation of international flights and the maintenance of social order, are in close contact with other personnel and face greater occupational risk exposure, leaving them susceptible to further waves of SARS-CoV-2 infection. Therefore, we explored the immunogenicity and safety of the COVD-19 inactivated vaccination schemes at three different intervals of either 14 days, 21 days or 28 days in high-risk occupational population to optimize the inactivated vaccination regimen. We would continue to follow up until months 3,6 , and 12 in the further study. 


\section{Methods}

\section{Study design and participants}

We conducted a randomized, controlled phase IV trial of the SARS-CoV-2 inactivated vaccine manufactured by Beijing Biological Products Institute Co., Ltd. between January and May 2021 in Taiyuan City, Shanxi Province, China. Written informed consents were obtained from all participants before enrollment. Eligible participants were airport ground staff and public security officers aged 18-59 years, without previous SARS-CoV-2 vaccination and infection, and negative for SARS-CoV-2 nucleic acid. Exclusion criteria were participants with (1) history or family history of allergy, convulsion, epilepsy, encephalopathy or psychosis; (2) any intolerance or allergy to any component of the vaccine; (3) known or suspected diseases including severe respiratory disease, severe cardiovascular disease, severe liver or kidney disease, medically uncontrollable hypertension (systolic blood pressure $\geq 140 \mathrm{mmHg}$ and diastolic blood pressure $\geq 90 \mathrm{mmHg}$ ), complications of diabetes mellitus, malignancy, various acute diseases or acute episodes of chronic disease; (4) various infectious, suppurative and allergic skin diseases; congenital or acquired immunodeficiency; (5) other vaccination history within 14 days before vaccination; (6) a history of coagulation dysfunction, a history of non-specific immunoglobulin injection within 1 month prior to enrollment; acute illness with fever (body temperature $>37.0{ }^{\circ} \mathrm{C}$ ); and (7) being pregnant or breastfeeding.

The protocol was approved by the Ethics Committee of Shanxi Provincial Center for Disease Control (SXCDCIRBPJ2020056001) and Prevention and was conducted in accordance with the Declaration of Helsinki and Good Clinical Practice. All participants signed a consent form after being informed about the study. The trial was registered with ChiCTR.org.cn (ChiCTR2100041705, ChiCTR2100041706).

\section{Procedures}

A computerized random number generator performed block randomization with a randomly selected block size of 6, and eligible participants were randomly assigned into three groups to receive two doses inactivated SARSCoV-2 vaccine at the schedule of day $0-14$, day $0-21$, or day $0-28$. Each dose of vaccine containing $4 \mu \mathrm{g}$ of inactivated SARS-CoV-2 virus antigen was intramuscularly injected into the lateral deltoid muscle of the upper arm. The vaccines used in this study were inactivated vaccine (Vero Cell) produced by Beijing Biological Products Institute Co., Ltd. Demographic information [age, gender, body mass index (BMI), marital status, and education level], influenza vaccination history, smoking, drinking, and chronic diseases were collected via questionnaire investigation.

\section{Safety assessment}

After each dose was vaccinated, the participants were observed for any immediate reaction for $30 \mathrm{~min}$, and local and systemic adverse reactions were collected. Participants were required to record the local adverse events and systemic adverse events on diary cards within 7 days of each injection. Any other unsolicited symptoms were also recorded during a 28-day follow-up period after each injection by spontaneous report from the participants combined with the regular visit. The solicited adverse reactions included local reactions (pain, induration, swelling, rash, flush, and pruritus) and systematic reactions [fever, diarrhea, dysphagia, anorexia, vomiting, nausea, muscle pain (non-vaccination sites), arthralgia, headache, cough, dyspnea, skin and mucosal abnormalities, acute allergic reactions, and fatigue].

\section{Laboratory methods}

Oropharyngeal/nasal swabs were collected for detecting SARS-CoV-2 nucleic acid from all subjects by using reverse transcriptase-polymerase chain reaction (RTPCR) test to determine whether subjects have occurred SARS-COV-2 infection before the first, second dose of vaccine vaccination, and 28 days after the whole course of vaccination, respectively. Blood samples were taken from participants for serology tests before the first injection and on day 28 after the second injection. The neutralizing antibody to live SARS-CoV-2 [strain $19 \mathrm{nCoV}-\mathrm{CDC}$ Tan-Strain 05 (QD01)] were quantified using a micro cytopathogenic effect assay at baseline and 28 days after immunization. A positive antibody response (seroconversion) was defined as post-injection titer of at least 1:16 if the baseline titer was below 1:4, or at least a fourfold increase in post-injection titer from baseline if the baseline titer was at least 1:4 [21]. We defined the neutralizing antibody seroconversion rate as post-injection titer of a 16-fold (Baseline titers were all below 1:4).

\section{Outcomes}

The primary immunogenic endpoints were the seroconversion rates [geometric mean titer $(\mathrm{GMT}) \geq 16$ ] and GMT of neutralizing antibody to live SARS-CoV-2 at day 28 after the last dose. Secondary immunogenic endpoints were the positive rates (GMT $\geq 32,64,128,256) 28$ days after the whole course of vaccination, respectively. The primary endpoint for safety was the occurrence of adverse reactions within 7 days after the first and second vaccinations. Adverse events within 28 days after the first and the second vaccinations across the three groups were 
analyzed as secondary safety endpoints. Figure 1 shows the study protocol.

\section{Statistical analysis}

The study sample size of 360 participants provided $84.4 \%$ power to detect a difference of $5 \%$ ( $85 \%$ vs $80 \%)$ of responders in the $0-21$ and $0-28$ vaccination groups compared with the $0-14$ group, respectively in airport ground staff and public security officers. Data were recorded using EpiData version 3.1 (EpiData Association, Odense, Denmark), and analyses were performed using SAS version 9.3 (SAS Institute, Cary, NC, USA). Analysis of variance (ANOVA) was used to analyze continuous data, and the chi-square or Fisher's exact test was used for categorical data. We assessed immunogenic endpoints by the intention-to-treat (ITT) analysis (i.e., subjects who undertake randomization) and per-protocol (PP) analysis (i.e., subjects who compliant to the protocol, receive 2 doses of vaccine according to the requirements of the protocol, and have serum-testing results before and after immunization). Multinomial logistic regression analysis and unconditional logistic regression model were used to determine the influencing factors of SARS-CoV-2 neutralizing antibody immunization. The safety analysis was performed on data from all subjects who received vaccination after randomization. The level of statistical significance for all analyses was $P<0.05$.

\section{Results}

\section{Study participants and baseline characteristics}

Between January and May 2021, 810 participants were screened, and 809 were enrolled $(73.2 \%$ male, $26.8 \%$ female; mean age 38.8 years). Of the 809 participants who were enrolled, 405 participants were the public security officers and 404 participants were the airport ground staff; with 270, 270, and 269 participants in the group
$0-14,0-21,0-28$ vaccination cohort. All enrolled participants received the first injection and completed the twodose vaccination schedule. A total of 256, 247 and 241 patients in the $0-14,0-21$, and $0-28$ groups, respectively, completed the follow-up 28 days after the whole course of vaccination (Fig. 2). The baseline characteristics of the participants are shown in Table 1. There were no significant differences in demographic and behavioral characteristics among the three groups at baseline and 28 days after the whole course of vaccination $(P>0.05$; Table 1 , Additional file 1: Supplement 1).

\section{Assessment of immunity elicited by the vaccine in the three immunization procedures \\ Seroconversion rate and GMT of SARS-CoV-2 neutralizing antibody in the three groups}

By day 28 after the second injection, the seroconversion rates of neutralizing antibody $(\mathrm{GMT} \geq 16)$ were all $100.0 \%$ in the $0-14,0-21$, and $0-28$ groups. SARSCoV-2 neutralizing antibody with a GMT of 98.4 (95\% CI: 88.4-108.4) was noted in the 0-14 group, which was significantly lower compared with 134.4 (95\% CI: $123.1-$ $145.7)$ in the $0-21$ group $(P<0.001$ vs $0-14$ group $)$ and 145.5 (95\% CI: $131.3-159.6)$ in the $0-28$ group $(P<0.001$ vs $0-14$ group). The ITT analysis showed similar results that the GMT were between 93.5 and 129.8 in the three groups.

Then we used different criteria to determine the immunization of neutralizing antibody for comparison. The positive rates of neutralizing antibody GMT $\geq 32$ were 86.7\% (222/256), 96.4\% (238/247; $P<0.001)$ and $95.9 \%$ $(231 / 241 ; P<0.001)$ in the $0-14,0-21$, and $0-28$ groups, respectively. The positive rates of neutralizing antibody GMT $\geq 64,128$, and 256 were $60.9 \%$ (156/256), 31.6\% $(81 / 256)$ and $9.0 \%(23 / 256)$ in the $0-14$ group, $84.6 \%$ $(209 / 247), 55.9 \%(138 / 247)$ and $15.0 \%(37 / 247)$ in the

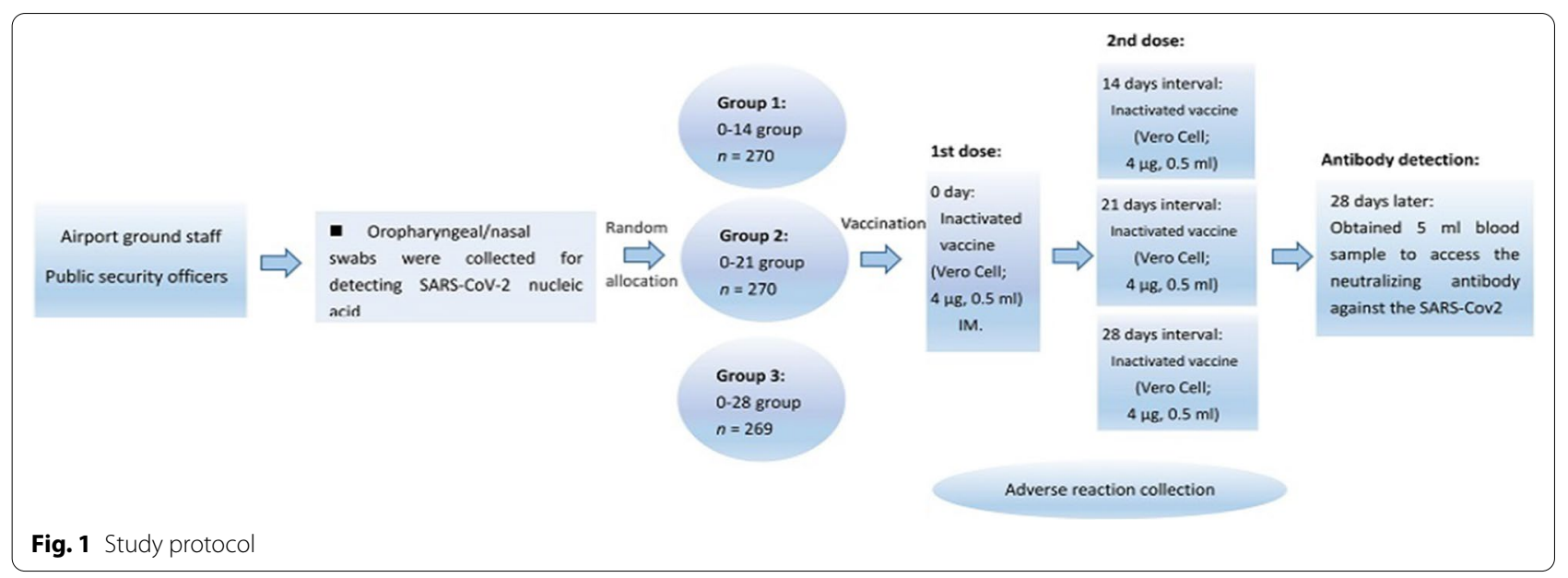




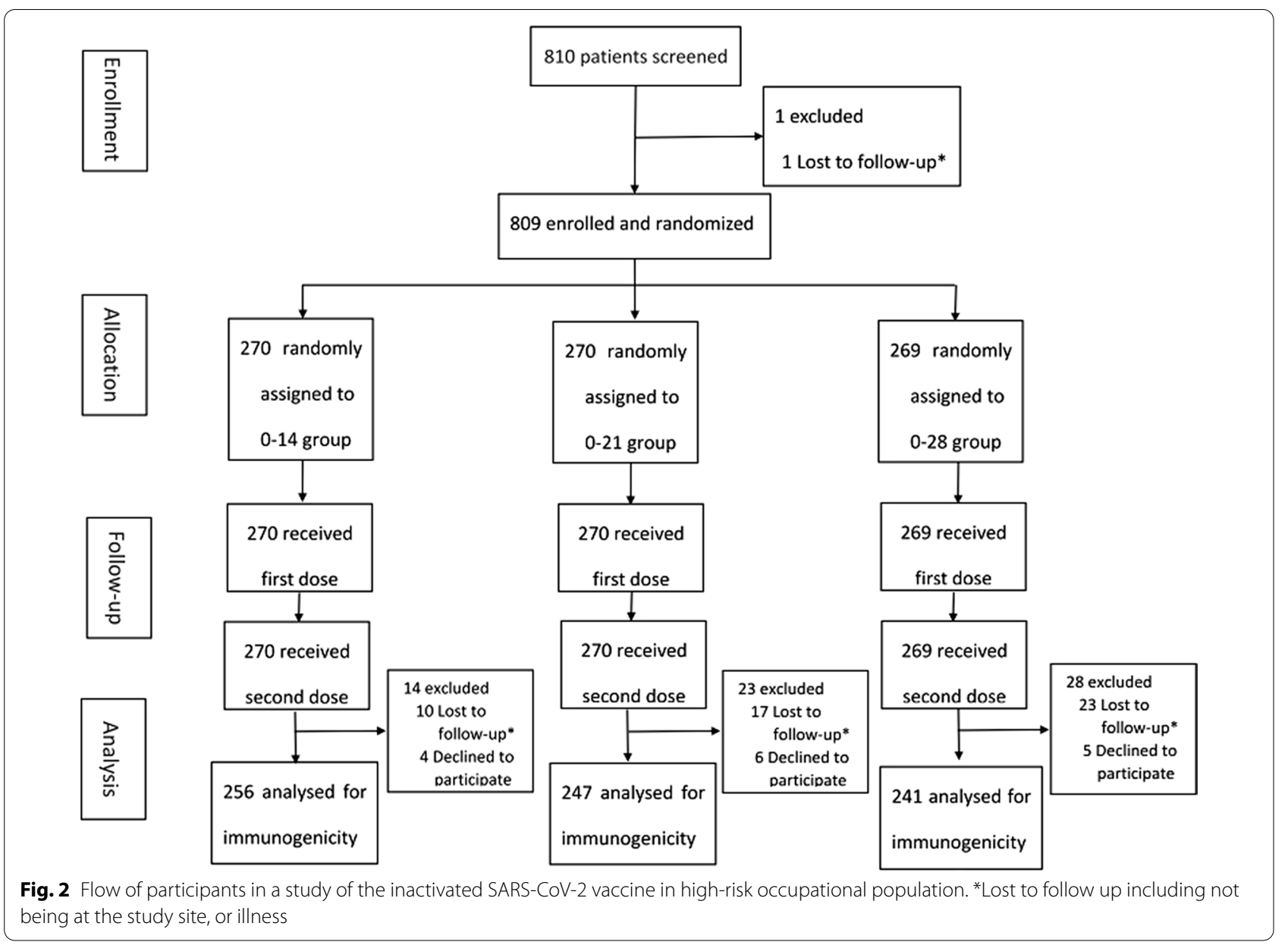

0-21 group, 80.9\% (195/241), 51.5\% (124/241) and 17.4\% $(42 / 241)$ in the $0-28$ group, respectively (Table 2$)$. The positive rates of neutralizing antibody (GMT $\geq 32,64$, 128 , or 256 ) in the $0-21$ and $0-28$ groups were significantly higher than that in the $0-14$ group. The ITT analysis yielded similar results (Table 2).

\section{Distribution of SARS-CoV-2 neutralizing antibody in the three groups}

In the 0-14 group, the proportion of neutralizing antibody was higher at the GMT 32-63 $(25.8 \%, 66 / 256)$ and 64-127 (29.3\%, 75/256), while the proportion of neutralizing antibody in the $0-21$ and $0-28$ groups was higher at the GMT of $64-127(28.7 \%, 71 / 247 ; 29.5 \%, 71 / 241)$ and 128-255 (40.9\%, 101/247; 34.0\%, 82/241), respectively (Fig. 3). There was a significant difference in distribution of SARS-CoV-2 neutralizing antibody GMT among the three vaccination groups $(P<0.001)$.

\section{Stratified analysis}

In the further analysis stratified by age and gender, we observed the similar results that the GMT of
SARS-CoV-2 neutralizing antibody and positive rates in the $0-21$ and $0-28$ groups were superior to the $0-14$ group for the participants with different characteristic levels (Additional file 1: Supplement 2, Supplement 3). And the similar distribution results of SARS-CoV-2 neutralizing antibody were observed for the three groups (Additional file 1: Supplement 4).

\section{Influencing factors of SARS-CoV-2 neutralizing antibody immunization by multinomial logistic regression}

Multinomial logistic regression was performed to examine the influencing factors of SARS-CoV-2 neutralizing antibody immunization depending on their extent (GMT: $16-63=1$, GMT: $64-127=2$, GMT: $\geq 128=3$, ref $=1$ ) and the results showed that only the vaccination regimen was associated with the antibody response. After adjusting for age, gender, BMI, marital status, education level, influenza vaccination history, smoking, drinking, and chronic diseases, participants who received 0-21 vaccination regimen was 2.5 times higher than the $0-14$ vaccination group when GMT was 64-127 (95\% CI: 1.5-4.1), and it was 4.4-fold higher than that in $0-14$ 
Table.1 Baseline characteristics of high-risk occupational population with different vaccinations

\begin{tabular}{|c|c|c|c|c|c|}
\hline Characteristics & $\begin{array}{l}\text { Total } \\
(n=809)\end{array}$ & $\begin{array}{l}\text { 0-14 group } \\
(n=270)\end{array}$ & $\begin{array}{l}0-21 \text { group } \\
(n=270)\end{array}$ & $\begin{array}{l}0-28 \text { group } \\
(n=269)\end{array}$ & $P$ \\
\hline Gender & & & & & 0.449 \\
\hline Male & $592(73.2)$ & $192(71.1)$ & $196(72.6)$ & $204(75.8)$ & \\
\hline Female & $217(26.8)$ & 78 (28.9) & $74(27.4)$ & $65(24.2)$ & \\
\hline Age (years) & & & & & 0.229 \\
\hline$<40$ & $463(57.2)$ & $144(53.3)$ & $156(57.8)$ & $163(60.6)$ & \\
\hline$\geq 40$ & $346(42.8)$ & $126(46.7)$ & $114(42.2)$ & $106(39.4)$ & \\
\hline Education level & & & & & 0.335 \\
\hline Junior high school or lower & $74(9.1)$ & $31(11.5)$ & $25(9.3)$ & $18(6.7)$ & \\
\hline Senior high school & $37(4.6)$ & $10(3.7)$ & $12(4.4)$ & $15(5.6)$ & \\
\hline College or higher & $698(86.3)$ & $229(84.8)$ & $233(86.3)$ & $236(87.7)$ & \\
\hline Ethnicity & & & & & $0.366^{\mathrm{a}}$ \\
\hline Han ethnicity & 797 (98.5) & $268(99.3)$ & $264(97.8)$ & $265(98.5)$ & \\
\hline Other & $12(1.5)$ & $2(0.7)$ & $6(2.2)$ & $4(1.5)$ & \\
\hline Marital status & & & & & 0.267 \\
\hline Married & $621(76.8)$ & $217(80.4)$ & $196(72.6)$ & $208(77.3)$ & \\
\hline Unmarried & $165(20.4)$ & $47(17.4)$ & $66(24.4)$ & $52(19.3)$ & \\
\hline Divorced or widowed & $23(2.8)$ & $6(2.2)$ & $8(3.0)$ & $9(3.4)$ & \\
\hline $\mathrm{BMI}\left(\mathrm{kg} / \mathrm{m}^{2}\right)$ & & & & & 0.848 \\
\hline$<18.5$ & $19(2.3)$ & $8(3.0)$ & $6(2.2)$ & $5(1.8)$ & \\
\hline $18.5-$ & $333(41.2)$ & $110(40.7)$ & $116(43.0)$ & 107 (39.8) & \\
\hline$\geq 24$ & $457(56.5)$ & $152(56.3)$ & $148(54.8)$ & $157(58.4)$ & \\
\hline Influenza vaccination history & & & & & 0.865 \\
\hline No & $549(67.9)$ & $180(66.7)$ & $184(68.2)$ & $185(68.8)$ & \\
\hline Yes & $260(32.1)$ & $90(33.3)$ & $86(31.8)$ & $84(31.2)$ & \\
\hline Occupation & & & & & 0.991 \\
\hline Public security officers & $405(50.1)$ & $135(50.0)$ & $136(50.4)$ & $134(49.8)$ & \\
\hline Airport ground staff & $404(49.9)$ & $135(50.0)$ & $134(49.6)$ & $135(50.2)$ & \\
\hline Smoking & & & & & 0.368 \\
\hline No & $545(67.4)$ & $190(70.4)$ & $181(67.0)$ & $174(64.7)$ & \\
\hline Yes & $264(32.6)$ & $80(29.6)$ & $89(33.0)$ & $95(35.3)$ & \\
\hline Drinking & & & & & 0.906 \\
\hline No & $621(76.8)$ & $206(76.3)$ & $206(76.3)$ & $209(77.7)$ & \\
\hline Yes & $188(23.2)$ & $64(23.7)$ & $64(23.7)$ & $60(22.3)$ & \\
\hline Chronic diseases & & & & & 0.978 \\
\hline No & $754(93.2)$ & $252(93.3)$ & $252(93.3)$ & $250(92.9)$ & \\
\hline Yes & $55(6.8)$ & $18(6.7)$ & $18(6.7)$ & $19(7.1)$ & \\
\hline
\end{tabular}

Results expressed as $n$ (\%)

${ }^{a}$ Fisher's exact test

vaccination group at GMT $\geq 128$ (95\% CI: 2.8-7.1). Participants in the $0-28$ group had 2.0-fold odds when GMT was $64-127$ (95\% CI 1.2-3.3) than that in the $0-14$ group and had 3.3-fold odds of GMT $\geq 128$ (95\% CI: 2.1-5.2) (Table 3).
Logistic regression analysis of factors influencing the SARS-CoV-2 neutralizing antibody immunization Logistic regression analysis results showed that only vaccination regimen was associated with SARS-CoV-2 neutralizing antibody immunization. After adjusting for age, gender, BMI, marital status, education level, influenza vaccination history, smoking, drinking, and chronic diseases, the results showed that the participants in $0-21$ and $0-28$ groups were 4.1 (95\% CI: 1.9-8.8) and 3.6 (95\% 
Table.2 The seroconversion rate and GMT of SARS-CoV-2 neutralizing antibody in the three groups

\begin{tabular}{|c|c|c|c|c|c|c|}
\hline \multirow[t]{2}{*}{ SARS-CoV-2 neutralizing antibody } & \multicolumn{3}{|c|}{ Per-protocol analysis } & \multicolumn{3}{|c|}{ Intention-to-treat analysis } \\
\hline & $\begin{array}{l}\text { 0-14 group } \\
(n=256)\end{array}$ & $\begin{array}{l}0-21 \text { group } \\
(n=247)\end{array}$ & $\begin{array}{l}0-28 \text { group } \\
(n=241)\end{array}$ & $\begin{array}{l}\text { 0-14 group } \\
(n=270)\end{array}$ & $\begin{array}{l}0-21 \text { group } \\
(n=270)\end{array}$ & $\begin{array}{l}\text { 0-28 group } \\
(n=269)\end{array}$ \\
\hline \multicolumn{7}{|l|}{ Primary analysis } \\
\hline GMT $(95 \%$ Cl) & $\begin{array}{l}98.4 \\
(88.4-108.4)^{a}\end{array}$ & $\begin{array}{l}134.4 \\
(123.1-145.7)^{b}\end{array}$ & $\begin{array}{l}145.5 \\
(131.3-159.6)^{b}\end{array}$ & $\begin{array}{l}93.5 \\
(83.7-103.3)^{\mathrm{a}}\end{array}$ & $\begin{array}{l}122.0 \\
(110.7-133.2)^{b}\end{array}$ & $\begin{array}{l}129.8 \\
(116.2-143.4)^{b}\end{array}$ \\
\hline \multicolumn{7}{|l|}{ Seroconversion (GMT $\geq 16$ ) } \\
\hline No, $n(\%)$ & $0(0.0)$ & $0(0.0)$ & $0(0.0)$ & $14(5.2)$ & $23(8.5)$ & $28(10.4)$ \\
\hline Yes, $n(\%)$ & $256(100.0)$ & $247(100.0)$ & $241(100.0)$ & $256(94.8)^{\mathrm{a}}$ & $247(91.5)^{\mathrm{ab}}$ & $241(89.6)^{b}$ \\
\hline Crude OR (95\% Cl) & - & - & - & 1.0 & $0.6(0.3-1.2)$ & $0.5(0.2-0.9)$ \\
\hline Adjusted OR (95\% Cl) & - & - & - & 1.0 & $0.6(0.3-1.2)$ & $0.5(0.3-1.0)$ \\
\hline \multicolumn{7}{|l|}{ Secondary analysis } \\
\hline \multicolumn{7}{|l|}{$\mathrm{GMT} \geq 32$} \\
\hline No, $n(\%)$ & $34(13.3)$ & $9(3.6)$ & $10(4.1)$ & $48(17.8)$ & $32(11.8)$ & $38(14.1)$ \\
\hline Yes, $n(\%)$ & $222(86.7)^{\mathrm{a}}$ & $238(96.4)^{b}$ & $231(95.9)^{\mathrm{b}}$ & $222(82.2)$ & $238(88.2)$ & $231(85.9)$ \\
\hline Crude $O R(95 \% C l)$ & 1.0 & $4.1(1.9-8.6)$ & $3.5(1.7-7.3)$ & 1.0 & $1.6(1.0-2.6)$ & $1.3(0.8-2.1)$ \\
\hline Adjusted OR (95\% Cl) & 1.0 & $4.1(1.9-8.8)$ & $3.6(1.7-7.6)$ & 1.0 & $1.7(1.0-2.7)$ & $1.4(0.8-2.2)$ \\
\hline \multicolumn{7}{|l|}{$\mathrm{GMT} \geq 64$} \\
\hline No, $n(\%)$ & $100(39.1)$ & $38(15.4)$ & $46(19.1)$ & $114(42.2)$ & $61(22.6)$ & $74(27.5)$ \\
\hline Yes, $n(\%)$ & $156(60.9)^{a}$ & $209(84.6)^{b}$ & $195(80.9)^{b}$ & $156(57.8)^{\mathrm{a}}$ & $209(77.4)^{b}$ & $195(72.5)^{b}$ \\
\hline Crude OR (95\% Cl) & 1.0 & $3.5(2.3-5.4)$ & $2.7(1.8-4.1)$ & 1.0 & $2.5(1.7-3.6)$ & $1.9(1.3-2.8)$ \\
\hline Adjusted OR (95\% Cl) & 1.0 & $3.5(2.7-5.8)$ & $2.7(1.8-4.1)$ & 1.0 & $2.5(1.7-3.7)$ & $1.9(1.4-2.8)$ \\
\hline \multicolumn{7}{|l|}{$\mathrm{GMT} \geq 128$} \\
\hline No, $n(\%)$ & $175(68.4)$ & $109(44.1)$ & $117(48.5)$ & $189(70.0)$ & $132(48.9)$ & $145(53.9)$ \\
\hline Yes, $n(\%)$ & $81(31.6)^{\mathrm{a}}$ & $138(55.9)^{b}$ & $124(51.5)^{b}$ & $81(30.0)^{\mathrm{a}}$ & $138(51.1)^{b}$ & $124(46.1)^{b}$ \\
\hline Crude OR (95\% Cl) & 1.0 & $2.7(1.9-3.9)$ & $2.3(1.6-3.3)$ & 1.0 & $2.44(1.7-3.5)$ & $2.0(1.4-2.8)$ \\
\hline Adjusted OR (95\% Cl) & 1.0 & $2.7(1.9-3.9)$ & $2.3(1.6-3.4)$ & 1.0 & $2.43(1.7-3.5)$ & $2.1(1.4-3.0)$ \\
\hline \multicolumn{7}{|l|}{$\mathrm{GMT} \geq 256$} \\
\hline No, $n(\%)$ & $233(91.0)$ & $210(85.0)$ & $199(82.6)$ & $247(91.5)$ & $233(86.3)$ & $227(84.4)$ \\
\hline Yes, $n(\%)$ & $23(9.0)^{\mathrm{a}}$ & $37(15.0)^{b}$ & $42(17.4)^{b}$ & $23(8.5)^{\mathrm{a}}$ & $37(13.7)^{a b}$ & $42(15.6)^{b}$ \\
\hline Crude OR (95\% Cl) & 1.0 & $1.8(1.0-3.1)$ & $2.1(1.2-3.7)$ & 1.0 & $1.7(1.0-3.0)$ & $2.0(1.2-3.4)$ \\
\hline Adjusted OR (95\% Cl) & 1.0 & $1.8(1.0-3.2)$ & $2.3(1.3-4.0)$ & 1.0 & $1.8(1.0-3.1)$ & $2.1(1.2-3.6)$ \\
\hline
\end{tabular}

- No result value

GMT Geometric mean titer; Cl Confidence interval; OR Odds ratio

a,b There was significant difference with the different letters

CI: $1.7-7.6)$ times more likely to be positive (GMT $\geq 32$ ) than those in 0-14 groups, respectively; and the participants in 0-21 and 0-28 groups showed higher positive rates than those in $0-14$ group (GMT $\geq 64$ ) (OR: 3.5, 95\% CI: 2.3-5.4; OR: 2.7, 95\% CI: 1.8-4.1). The similar results were found at GMT $\geq 128$ or 256 (Table 4).

\section{Safety outcomes}

The overall incidence of adverse reactions was $4.1 \%$ $(11 / 270), 4.8 \%(13 / 270)$, and $3.7 \%(10 / 269)$ in the $0-14$, $0-21$ and $0-28$ vaccination cohort group.

Solicited adverse reactions were reported by $8(3.0 \%)$ in the $0-14$ vaccination cohort group, $11(4.1 \%)$ in the $0-21$ vaccination cohort group, and $7(2.6 \%)$ in the $0-28$ vaccination cohort group within 7 days after injection. No significant differences were found in the occurrence of solicited and unsolicited adverse reactions among the three groups. Pain, swelling, pruritus, diarrhea and fatigue within 7 days after vaccination were reported by 4 (1.5\%), $2(0.7 \%), 1(0.4 \%), 0(0.0 \%)$, and $1(0.4 \%)$ subject in the $0-14$ group, 7 (2.6\%), $0(0.0 \%), 2(0.7 \%), 1$ $(0.4 \%)$, and $1(0.4 \%)$ subject in the $0-21$ group, and 2 $(0.7 \%), 2(0.7 \%), 0(0.0 \%), 1(0.4 \%)$, and $2(0.7 \%)$ subject in the $0-28$ vaccination group, respectively. Rash, cough and headache within 28 days after vaccination were reported by $1(0.4 \%), 1(0.4 \%)$, and $1(0.4 \%)$ subject in the $0-14$ group, $1(0.4 \%), 1(0.4 \%)$, and $0(0.0 \%)$ subject in the $0-21$ group, and $1(0.4 \%), 1(0.4 \%)$, and 


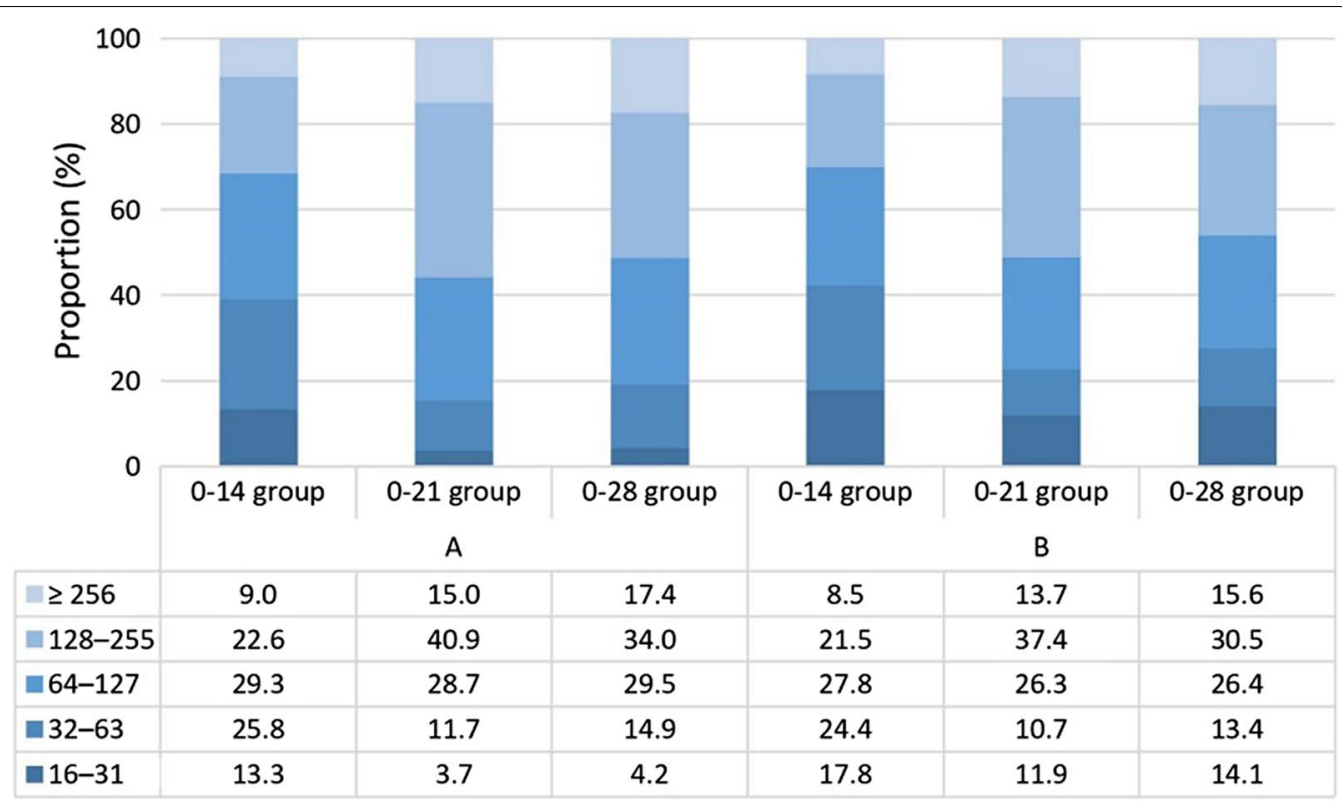

Fig. 3 Distribution of SARS-CoV-2 neutralizing antibody. A Per-protocol analysis, B intention-to-treat analysis. The table shows the percentages of SARS-CoV-2 neutralizing antibody in each group

Table.3 Influencing factors of SARS-CoV-2 neutralizing antibody immunization by multinomial logistic regression

\begin{tabular}{lll}
\hline Variables & OR $(\mathbf{9 5 \%} \mathrm{Cl})$ & OR $(\mathbf{9 5 \%} \mathbf{C l})$ \\
\hline GMT 64-127 (ref: 16-63) & & \\
0-14 group & 1.0 & 1.0 \\
0-21 group & $2.5(1.5-4.1)$ & $2.5(1.5-4.1)$ \\
0-28 group & $2.1(1.3-3.3)$ & $2.0(1.2-3.3)$ \\
GMT $\geq 128$ (ref: 16-63) & & \\
0-14 group & 1.0 & 1.0 \\
0-21 group & $4.5(2.8-7.1)$ & $4.4(2.8-7.1)$ \\
0-28 group & $3.3(2.1-5.2)$ & $3.3(2.1-5.2)$
\end{tabular}

$G M T$, Geometric mean titer; $C l$, confidence interval; OR: odds ratio

*Adjusted by age, gender, BMI, marital status, education level, influenza vaccination history, smoking, drinking, and chronic diseases

$1(0.4 \%)$ subject in the $0-28$ group, respectively. The reported adverse reactions did not differ significantly among the three study groups $(P>0.05)$. None of the subjects reported serious adverse reactions or became SARS-CoV-2 infected during the follow-up period (Table 5).

\section{Discussion}

Given the COVID-19 pandemic continuing to unfold, a safe and effective vaccine is necessary to contain the global COVID-19 pandemic and prevent further illness and fatalities. Inactivated vaccines are generally safe and
Table.4 Logistic regression analysis of factors influencing the SARS-COV-2 neutralizing antibody immunization

\begin{tabular}{lll}
\hline Variables & OR $(\mathbf{9 5} \% \mathrm{Cl})$ & $\mathbf{O R}(\mathbf{9 5} \% \mathbf{C l})^{*}$ \\
\hline GMT $\geq 32$ & & 1.0 \\
$0-14$ group & 1.0 & $4.1(1.9-8.8)$ \\
$0-21$ group & $4.1(1.9-8.6)$ & $3.6(1.7-7.6)$ \\
$0-28$ group & $3.5(1.7-7.3)$ & \\
GMT $\geq 64$ & & 1.0 \\
$0-14$ group & 1.0 & $3.5(2.3-5.4)$ \\
$0-21$ group & $3.5(2.3-5.4)$ & $2.7(1.8-4.1)$ \\
$0-28$ group & $2.7(1.8-4.1)$ & \\
GMT $\geq 128$ & & 1.0 \\
$0-14$ group & 1.0 & $2.7(1.9-3.9)$ \\
$0-21$ group & $2.7(1.9-3.9)$ & $2.3(1.6-3.4)$ \\
$0-28$ group & $2.3(1.6-3.3)$ & 1.0 \\
GMT $\geq 256$ & & $1.8(1.0-3.2)$ \\
$0-14$ group & 1.0 & $2.3(1.3-4.0)$ \\
$0-21$ group & $1.8(1.0-3.1)$ & \\
$0-28$ group & $2.1(1.2-3.7)$ &
\end{tabular}

GMT geometric mean titer; $\mathrm{Cl}$ confidence interval; $\mathrm{OR}$ odds ratio

*Adjusted by age, gender, BMI, marital status, education level, influenza vaccination history, smoking, drinking, and chronic diseases

widely used for prevention of infectious diseases. The airport ground staff and public security officers are in close contact with other personnel and face greater occupational risk exposure to SARS-CoV-2 infection. Although 
Table.5 Summary of solicited and unsolicited adverse reactions occurred within 28 days during the study period

\begin{tabular}{|c|c|c|c|c|}
\hline Adverse reaction $^{*}$ & $\begin{array}{l}0-14 \text { group } \\
(n=270)\end{array}$ & $\begin{array}{l}0-21 \text { group } \\
(n=270)\end{array}$ & $\begin{array}{l}0-28 \text { group } \\
(n=269)\end{array}$ & $P$ \\
\hline \multicolumn{5}{|c|}{ Solicited adverse reactions within $0-7$ days } \\
\hline Local reactions & $7(2.6)$ & $9(3.3)$ & $4(1.5)$ & 0.418 \\
\hline Pain & $4(1.5)$ & $7(2.6)$ & $2(0.7)$ & 0.263 \\
\hline Swelling & $2(0.7)$ & $0(0.0)$ & $2(0.7)$ & 0.479 \\
\hline Pruritus & $1(0.4)$ & $2(0.7)$ & $0(0.0)$ & 0.777 \\
\hline Systemic reactions & $1(0.4)$ & $2(0.7)$ & $3(1.1)$ & 0.545 \\
\hline Diarrhea & $0(0.0)$ & $1(0.4)$ & $1(0.4)$ & 0.777 \\
\hline Fatigue & $1(0.4)$ & $1(0.4)$ & $2(0.7)$ & 0.702 \\
\hline \multicolumn{5}{|c|}{ Unsolicited adverse reactions within 8-28 days } \\
\hline Local reactions & $1(0.4)$ & $1(0.4)$ & $1(0.4)$ & 1.000 \\
\hline Rash & $1(0.4)$ & $1(0.4)$ & $1(0.4)$ & 1.000 \\
\hline Systemic reactions & $2(0.7)$ & $1(0.4)$ & $2(0.7)$ & 0.876 \\
\hline Cough & $1(0.4)$ & $1(0.4)$ & $1(0.4)$ & 1.000 \\
\hline Headache & $1(0.4)$ & $0(0.0)$ & $1(0.4)$ & 0.777 \\
\hline
\end{tabular}

Results expressed as $n$ (\%)

${ }^{*}$ Adverse reaction data only list the occurrence of this symptom

SARS-CoV-2 vaccines are widely administered in China or other countries, the optimal interval of injections remains unclear and there is lack of randomized controlled trials of inactivated SARS-CoV-2 vaccine in highrisk occupational population. This is the first randomized controlled trial for assessment of the immunogenicity and safety of an inactivated SARS-CoV-2 vaccine in highrisk occupational population.

Here, we explored the immunogenicity and safety of the three different SARS-CoV-2 inactivated vaccination schemes, and found that the GMT of neutralizing antibody were between 98.4 and 145.5, with the seroconversion rates $(\mathrm{GMT} \geq 16)$ being $100 \%$ in the three groups. The current clinical trials have also assessed the immunogenicity of SARS-CoV-2 vaccines, and have seen comparable results. The existing inactivated virus vaccines have shown significant immune responses (79-100\%) and neutralizing antibody titers $(18.9-282.7)[12,13$, $16,18-20]$. In addition, the efficacy of other kinds of SARS-CoV-2 vaccines in phase 3 clinical trials is approximately 70.4-95.0\% [22-25]. These studies indicated that the current SARS-CoV-2 vaccines have relatively good immunogenicity.

In our research, the SARS-CoV-2 neutralizing antibody titers of the $0-28,0-21$ groups were significantly greater than that of $0-14$ group. Xia et al. [12, 13] in both phase 1 and 2 found that a longer interval (21 days and 28 days) produced higher antibody responses compared with a shorter interval schedule (14 days) [GMT: 282.7 (221.2-361.4); 218.0 (181.8-261.3) vs 169.5 (132.2217.1)]. Similarly, Zhang et al. [16] and Pan et al. [18] found that the $0-28$ regimen of inactivated SARS-CoV-2 vaccine induced higher SARS-CoV-2 neutralizing antibody titers and seroconversion rates compared with the $0-14$ regimen. All of above studies indicated that longer interval schedule (0-21 regimen or $0-28$ regimen) of the inactivated SARS-CoV-2 vaccines may induce a better immunogenicity.

The incidence of adverse reactions in the $0-14,0-21$ and $0-28$ groups were similarly low. Moreover, we did not find severe adverse reaction, with the most common symptom being injection-site pain, indicating no safety concerns. The overall incidence of adverse events after vaccination was $3.7-4.8 \%$ in our vaccine-treated groups, which is noticeably lower than that of other SARS-CoV-2 vaccine platform candidates such as viral-vectored vaccines, DNA or RNA vaccines [25-30]. The safety profile of this vaccine in our study is also lower than that of other inactivated SARS-CoV-2 vaccines $[12,16]$, which may be related to different population characteristics, and minor adverse reactions that are not reported.

As the COVID-19 pandemic continues to spread, multiple SARS-COV-2 variants have emerged. Of these, the Delta variant has currently become the dominant strain of SARS-CoV-2, causing public concern around the world [31]. One study of 366 participants aged 18-59 years in Guangzhou found that the two-dose scheme of the inactivated vaccines yielded an overall vaccine effectiveness of $59.0 \%$ against the Delta variant infection in realworld settings [32]. A real-world study [33] by $\mathrm{Hu}$ et al. found that Delta variant-infected patients in Jiangsu who received two doses of inactivated vaccine had an $88 \%$ 
reduced risk in progressing to the severe stage. Another real-world study [34] in Guangdong also showed that the effectiveness of full inactivated COVID-19 vaccination against COVID-19 pneumonia and severe illness caused by the B.1.617.2 (Delta) variant was $69.5 \%$ and $100.0 \%$ respectively. These studies indicated that the inactivated vaccines were effective against the Delta variant. Our study only tested the traditional SARS-CoV-2 strain which is one of our limitations.

In addition, our study had several limitations. First, since the majority of public security officers are male, there may be insufficient representation of the population. And we only reported immune response data for the high-risk occupational population aged 18 to 59 years. Further studies are required to assess the immunogenicity of inactivated SARS-CoV-2 vaccine in various populations, including general population, older people, children and adolescents. Second, data on long-term immunogenicity is not yet available, and the ongoing trial will provide more information. Third, cellular immunity and immune memory were not measured in the current study which need to be further studied.

\section{Conclusions}

In summary, a two-dose of inactivated SARS-CoV-2 vaccine at $0-21$ days and $0-28$ days regimens significantly improved SARS-CoV-2 neutralizing antibody level compared to the $0-14$ days regimen in high-risk occupational population, with seroconversion rates of $100.0 \%$, which to some extent provided a basis for optimizing the immunization strategy of inactivated vaccine against COVID19 among high-risk occupational population. The results were interim and the long-term immunogenicity and actual protection needs further study.

\section{Abbreviations \\ SARS-CoV-2: Severe acute respiratory syndrome coronavirus 2; COVID-19: Coronavirus disease 2019; GMT: Geometric mean titer; Cl: Confidence interval; ITT: Intention-to-treat; PP: Per-protocol; ARDS: Acute respiratory distress syndrome; BMI: Body mass index; RT-PCR: Reverse transcriptase-polymerase chain reaction; CDC: Center for Disease Control and Prevention; WHO: World Health Organization.}

\section{Supplementary Information}

The online version contains supplementary material available at https://doi. org/10.1186/s40249-021-00924-2.

Additional file 1: Supplement 1. Demographic and Behavioral Characteristics of High-risk Occupational Population 28 Days after the Whole Course of Vaccination. Supplement 2. SARS-CoV-2 Neutralizing Antibody Immunization 28 Days after the Whole Course of Vaccination Stratified by Age and Gender. b. SARS-CoV-2 neutralizing antibody immunization by gender. Supplement 3. GMT of SARS-CoV-2 Neutralizing Antibody 28 Days after the Whole Course of Vaccination Stratified by Age and Gender. Supplement 4. Distribution of SARS-CoV-2 Neutralizing Antibody 28 Days after the Whole Course of Vaccination Stratified by Age and Gender

\section{Acknowledgements}

This research was supported by the COVID-19 Project of Shanxi Provincial Finance, and the Project of Shanxi Provincial Key Laboratory for major infectious disease response. Thanks for gratefully all participants taking part in this research. We gratefully acknowledge the contribution from our colleagues and students, and staff members of the Shanxi Provincial Center for Disease Control and Outpatient Department of Shanxi Aviation Industry Group Co. LTD.

\section{Authors' contributions}

YF: conceptualization, conceived and developed the overall study design, reviewed and interpreted data, investigated and performed experiments, and drafted the manuscript. JC, TY: conceptualization, conceived and designed the study, analyzed the data, reviewed and interpreted data, investigated and performed experiments. YC: analyzed the data, reviewed and interpreted data, investigated and performed experiments. $X \mathrm{~L}, \mathrm{RX}, \mathrm{HL}, \mathrm{RX}, \mathrm{XZ}, \mathrm{ZW}, \mathrm{SM}$, and $\mathrm{LL}$ : investigated and performed experiments. LF* \& SW*: conceptualization, conceived and designed the study, reviewed and interpreted data, writing-review \& editing. All authors read and approved the final manuscript.

\section{Funding}

The study was supported by the COVID-19 Project of Shanxi Provincial Finance, and the Project of Shanxi Provincial Key Laboratory for major infectious disease response.

\section{Availability of data and materials}

The full study protocol and the datasets, which includes all data fields reported in this study, are available, following manuscript publication, upon request from the corresponding author (Professor Suping Wang, supingwang@sxmu.edu.cn), following the provision of ethics approval.

\section{Declarations}

\section{Ethics approval and consent to participate}

The study was approved by the Ethics Committee of Shanxi Provincial Center for Disease Control (SXCDCIRBPJ2020056001) and Prevention and the Chinese Clinical Trial Registry (ChiCTR2100041705, ChiCTR2100041706).

\section{Consent for publication}

Not applicable.

\section{Competing interests}

All authors declare that they have no competing interests.

\section{Author details}

${ }^{1}$ Department of Epidemiology, School of Public Health, Shanxi Medical University, 56 Xinjian South Road, Taiyuan 030001, Shanxi Province, China. ${ }^{2}$ Center of Clinical Epidemiology and Evidence Based Medicine, Shanxi Medical University, Taiyuan, China. ${ }^{3}$ Shanxi Provincial Center for Disease Control and Prevention, 8 Xiaonanguan Street, Taiyuan 030012, Shanxi Province, China. ${ }^{4}$ Shanxi Provincial Key Laboratory for Major Infectious Disease Response, Taiyuan, China. ${ }^{5}$ Outpatient Department of Shanxi Aviation Industry Group Co. LTD, Taiyuan, China.

Received: 20 August 2021 Accepted: 15 December 2021

Published online: 22 December 2021

\section{References}

1. World Health Organization. WHO Coronavirus (COVID-19) Dashboard. 2021. https://covid19.who.int/. Accessed 26 October 2021.

2. Chan JF, Yuan S, Kok KH, To KK, Chu H, Yang J, et al. A familial cluster of pneumonia associated with the 2019 novel coronavirus indicating person-to-person transmission: a study of a family cluster. Lancet. 2020;395(10223):514-23.

3. Chen N, Zhou M, Dong X, Qu J, Gong F, Han Y, et al. Epidemiological and clinical characteristics of 99 cases of 2019 novel coronavirus pneumonia in Wuhan, China: a descriptive study. Lancet. 2020;395(10223):507-13. 
4. Murdin AD, Barreto L, Plotkin S. Inactivated poliovirus vaccine: past and present experience. Vaccine. 1996;14(8):735-46.

5. Vellozzi C, Burwen DR, Dobardzic A, Ball R, Walton K, Haber P. Safety of trivalent inactivated influenza vaccines in adults: background for pandemic influenza vaccine safety monitoring. Vaccine. 2009;27(15):2114-20.

6. Gao Q, Bao L, Mao H, Wang L, Xu K, Yang M, et al. Development of an inactivated vaccine candidate for SARS-CoV-2. Science. 2020;369(6499):77-81.

7. Wang ZJ, Zhang HJ, Lu J, Xu KW, Peng C, Guo J, et al. Low toxicity and high immunogenicity of an inactivated vaccine candidate against COVID19 in different animal models. Emerg Microbes Infect. 2020;9(1):2606-18.

8. Wang $H$, Zhang $Y$, Huang B, Deng W, Quan $Y$, Wang W, et al. Development of an inactivated vaccine candidate, BBIBP-CorV, with potent protection against SARS-CoV-2. Cell. 2020;182(3):713-21.

9. Wu Z, Hu Y, Xu M, Chen Z, Yang W, Jiang Z, et al. Safety, tolerability, and immunogenicity of an inactivated SARS-CoV-2 vaccine (CoronaVac) in healthy adults aged 60 years and older: a randomised, doubleblind, placebo-controlled, phase 1/2 clinical trial. Lancet Infect Dis. 2021;21(6):803-12.

10. Ella R, Vadrevu KM, Jogdand H, Prasad S, Reddy S, Sarangi V, et al. Safety and immunogenicity of an inactivated SARS-CoV-2 vaccine, BBV152: a double-blind, randomised, phase 1 trial. Lancet Infect Dis. 2021;21(5):637-46.

11. Zhao J, Zhao S, Ou J, Zhang J, Lan W, Guan W, et al. COVID-19: coronavirus vaccine development updates. Front Immunol. 2020;11:602256.

12. Xia S, Duan K, Zhang Y, Zhao D, Zhang H, Xie Z, et al. Effect of an inactivated vaccine against SARS-CoV-2 on safety and immunogenicity outcomes: interim analysis of 2 randomized clinical trials. JAMA. 2020;324(10):951-60.

13. Xia S, Zhang Y, Wang Y, Wang H, Yang Y, Gao GF, et al. Safety and immunogenicity of an inactivated SARS-CoV-2 vaccine, BBIBP-CorV: a randomised, double-blind, placebo-controlled, phase 1/2 trial. Lancet Infect Dis. 2021;21(1):39-51.

14. The conditional approval by China National Medical Products Administrationfor the application registration of Novel Coronavirus inactivated vaccine (Vero cells) of Beijing Institute of Biological Products Co., Ltd. https://www.nmpa.gov.cn/yaowen/ypjgyw/20201231193329157.html.

15. Emergency Use Listing Procedure of Coronavirus Disease (COVID-19). WHO. https://extranet.who.int/pqweb/vaccines/covid-19-vaccines.

16. Zhang Y, Zeng G, Pan H, Li C, Hu Y, Chu K, et al. Safety, tolerability, and immunogenicity of an inactivated SARS-CoV-2 vaccine in healthy adults aged 18-59 years: a randomised, double-blind, placebo-controlled, phase 1/2 clinical trial. Lancet Infect Dis. 2021;21 (2):181-92.

17. Ariamanesh $M$, Porouhan P, PeyroShabany B, Fazilat-Panah D, Dehghani $M$, Nabavifard $M$, et al. Immunogenicity and safety of the inactivated SARS-CoV-2 vaccine (BBIBP-CorV) in patients with malignancy. Cancer Invest. 2021. https://doi.org/10.1080/07357907.2021.1992420.

18. Pan HX, Liu JK, Huang BY, Li GF, Chang XY, Liu YF, et al. Immunogenicity and safety of a severe acute respiratory syndrome coronavirus 2 inactivated vaccine in healthy adults: randomized, double-blind, and placebo-controlled phase 1 and phase 2 clinical trials. Chin Med J. 2021;134(11):1289-98.

19. Che Y, Liu X, Pu Y, Zhou M, Zhao Z, Jiang R, et al. Randomized, doubleblinded and placebo-controlled phase II trial of an inactivated SARSCoV-2 vaccine in healthy adults. Clin Infect Dis. 2020. https://doi.org/10. 1093/cid/ciaa1703.

20. Pu J, Yu Q, Yin Z, Zhang Y, Li X, Yin $Q$, et al. The safety and immunogenicity of an inactivated SARS-CoV-2 vaccine in Chinese adults aged 18-59 years: a phase I randomized, double-blinded, controlled trial. Vaccine. 2021;39(20):2746-54.

21. Al Kaabi N, Zhang Y, Xia S, Yang Y, Al Qahtani MM, Abdulrazzaq N, et al. Effect of 2 inactivated SARS-CoV-2 vaccines on symptomatic COVID-19 infection in adults: a randomized clinical trial. JAMA. 2021;326(1):35-45.

22. Polack FP, Thomas SJ, Kitchin N, Absalon J, Gurtman A, Lockhart S, et al. Safety and efficacy of the BNT162b2 mRNA COVID-19 vaccine. N Engl J Med. 2020;383(27):2603-15.

23. Logunov DY, Dolzhikova IV, Shcheblyakov DV, Tukhvatulin Al, Zubkova OV, Dzharullaeva AS, et al. Safety and efficacy of an rAd26 and rAd5 vector-based heterologous prime-boost COVID-19 vaccine: an interim analysis of a randomised controlled phase 3 trial in Russia. Lancet. 2021:397(10275):671-81.
24. Baden LR, El Sahly HM, Essink B, Kotloff K, Frey S, Novak R, et al. Efficacy and safety of the mRNA-1273 SARS-CoV-2 vaccine. N Engl J Med. 2021;384(5):403-16.

25. Voysey M, Clemens SAC, Madhi SA, Weckx LY, Folegatti PM, Aley PK, et al. Safety and efficacy of the ChAdOX1 nCoV-19 vaccine (AZD1222) against SARS-CoV-2: an interim analysis of four randomised controlled trials in Brazil, South Africa, and the UK. Lancet. 2021;397(10269):99-111.

26. Walsh EE, Frenck RW Jr, Falsey AR, Kitchin N, Absalon J, Gurtman A, et al. Safety and immunogenicity of two RNA-based COVID-19 vaccine candidates. T N Engl J Med. 2020;383(25):2439-50.

27. Jackson LA, Anderson EJ, Rouphael NG, Roberts PC, Makhene M, Coler $\mathrm{RN}$, et al. An mRNA vaccine against SARS-CoV-2 - preliminary report. New Engl J Med. 2020;383(20):1920-31.

28. Zhu FC, Guan XH, Li YH, Huang JY, Jiang T, Hou LH, et al. Immunogenicity and safety of a recombinant adenovirus type-5-vectored COVID-19 vaccine in healthy adults aged 18 years or older: a randomised, double-blind, placebo-controlled, phase 2 trial. Lancet. 2020;396(10249):479-88.

29. Zhu FC, Li YH, Guan XH, Hou LH, Wang WJ, Li JX, et al. Safety, tolerability, and immunogenicity of a recombinant adenovirus type-5 vectored COVID-19 vaccine: a dose-escalation, open-label, non-randomised, firstin-human trial. Lancet. 2020;395(10240):1845-54.

30. Mulligan MJ, Lyke KE, Kitchin N, Absalon J, Gurtman A, Lockhart S, et al. Phase I/II study of COVID-19 RNA vaccine BNT162b1 in adults. Nature. 2020;586(7830):589-93.

31. World Health Organization. Weekly epidemiological update on COVID-19 - 23 November 2021. https://www.who.int/publications/m/item/weeklyepidemiological-update-on-covid-19---23-november-2021. Accessed 23 Nov 2021.

32. Li XN, Huang $Y$, Wang $W$, Jing QL, Zhang $C H$, Qin PZ, et al. Effectiveness of inactivated SARS-CoV-2 vaccines against the Delta variant infection in Guangzhou: a test-negative case-control real-world study. Emerg Microbes Infect. 2021;10(1):1751-9.

33. Hu ZL, Tao BL, Li ZQ, Song Y, Yi CH, Li JW, et al. Effectiveness of inactive COVID-19 vaccines against severe illness in B.1.617.2 (Delta) variantinfected patients in Jiangsu, China. medRxiv. 2021:1-19

34. Kang M, Y Y Y, Li Y, Sun LM, Deng AP, Hu T, et al. Effectiveness of inactivated COVID-19 vaccines against COVID-19 pneumonia and severe illness caused by the B16172 (Delta) variant: evidence from an outbreak in Guangdong, China. https://doi.org/10.2139/ssrn.3895639

Ready to submit your research? Choose BMC and benefit from:

- fast, convenient online submission

- thorough peer review by experienced researchers in your field

- rapid publication on acceptance

- support for research data, including large and complex data types

- gold Open Access which fosters wider collaboration and increased citations

- maximum visibility for your research: over $100 \mathrm{M}$ website views per year

At BMC, research is always in progress.

Learn more biomedcentral.com/submissions 